\title{
eDGAR: a database of Disease-Gene Associations with annotated Relationships among genes
}

\author{
Giulia Babbi ${ }^{1}$, Pier Luigi Martelli ${ }^{1 *}$, Giuseppe Profiti ${ }^{1}$, Samuele Bovo ${ }^{1}$, Castrense Savojardo ${ }^{1}$ and Rita Casadio ${ }^{1,2}$ \\ From Varl-SIG 2016: identification and annotation of genetic variants in the context of structure, function, and disease \\ Orlando, Florida, USA. 09 July 2016
}

\begin{abstract}
Background: Genetic investigations, boosted by modern sequencing techniques, allow dissecting the genetic component of different phenotypic traits. These efforts result in the compilation of lists of genes related to diseases and show that an increasing number of diseases is associated with multiple genes. Investigating functional relations among genes associated with the same disease contributes to highlighting molecular mechanisms of the pathogenesis.

Results: We present eDGAR, a database collecting and organizing the data on gene/disease associations as derived from OMIM, Humsavar and ClinVar. For each disease-associated gene, eDGAR collects information on its annotation. Specifically, for lists of genes, eDGAR provides information on: i) interactions retrieved from PDB, BIOGRID and STRING; ii) co-occurrence in stable and functional structural complexes; iii) shared Gene Ontology annotations; iv) shared KEGG and REACTOME pathways; v) enriched functional annotations computed with NET-GE; vi) regulatory interactions derived from TRRUST; vii) localization on chromosomes and/or co-localisation in neighboring loci. The present release of eDGAR includes 2672 diseases, related to 3658 different genes, for a total number of 5729 gene-disease associations. 71\% of the genes are linked to 621 multigenic diseases and eDGAR highlights their common GO terms, KEGG/REACTOME pathways, physical and regulatory interactions. eDGAR includes a network based enrichment method for detecting statistically significant functional terms associated to groups of genes.

Conclusions: eDGAR offers a resource to analyze disease-gene associations. In multigenic diseases genes can share physical interactions and/or co-occurrence in the same functional processes. eDGAR is freely available at: edgar. biocomp.unibo.it
\end{abstract}

Keywords: Gene/disease relationship, Protein-protein interaction, Protein functional annotation, Functional enrichment

\section{Background}

The advent of fast and relatively costless techniques for genome screening boosts the research of genetic determinants of human phenotypes, with a specific focus on diseases [1]. By this, lists of genes involved in several diseases/phenotypes are available. One of the most comprehensive database of curated associations between human Mendelian disorders and genes is OMIM [2], collecting 4510 phenotypes with known molecular basis

\footnotetext{
* Correspondence: pierluigi.martelli@unibo.it

${ }^{1}$ Biocomputing Group, BiGeA, University of Bologna, Bologna, Italy Full list of author information is available at the end of the article
}

(release of May 2016). Updated resources of associations between variations and diseases are stored in the NCBIcurated ClinVar [3], the UniProt curated Humsavar list [4], and the commercial version of HGMD [5]. Integrative datasets, such as DisGeNet [6] and MalaCards [7] collect lists of gene-disease associations from different sources. MalaCards includes text mining of the scientific literature, gene annotations in terms of shared GO terms and associated pathways. DisGeNet integrates data of disease-associated genes and their variants. Furthermore, a database collecting data on digenic diseases (related to concomitant defects in pairs of genes) 
is available (DIDA, [8]) and reports the relationships between pairs of genes involved in 44 diseases.

As data accumulate, it emerges that an increasing number of diseases is associated with several genes. Independent or concomitant alterations in sequence or in expression of sets of genes are associated with the insurgence of genetically heterogeneous and polygenic diseases, respectively $[9,10]$. The scenario is even more complicated when different environmental and life-style related factors have strong influence on the insurgence and severity of the pathology [11]. The complex nature of the association between genes and diseases is one of the major challenges of Precision Medicine programs [12].

Dissecting the molecular mechanisms at the basis of the association between genotype and phenotype requires a deep investigation of the features shared among genes (or proteins) co-involved in the same disease. Indeed, by analyzing molecular features and functional interactions, important biological processes and pathways implicated in the disease can emerge and other genes possibly involved in interaction networks can be discovered $[13,14]$.

This work describes eDGAR, a database of gene-disease associations, supplemented with the annotations of intergenic relationships in heterogeneous and polygenic diseases. We merged, without redundancy, data from OMIM [2], ClinVar [3], and Humsavar [4]. Disease nomenclature derives from OMIM. OMIM phenotype entries are classified according to the OMIM Phenotypic Series, which cluster different entries related to identical or highly similar diseases associated with different genes. As compared to the above mentioned databases, our focus is on specific structural and functional annotations of the genes. For each gene, the database reports the cytogenetic location, links to the Ensembl [15], SwissProt [4] and PDB entries [16], Gene Ontology (GO) [17] annotations and to the KEGG and REACTOME pathways, when available. For sets of genes involved in the same disease, the database collects from publicly available databases different types of relationships: physical interactions, co-occurrence in protein complexes, regulatory interactions, shared functions and pathways, and co-localization in neighboring cytogenetic loci. A network - based approach (NET-GE $[18,19])$ provides statistical enrichment to functional terms. Information is organized in a relational database and an interface allows customized data search and retrieval.

The database is freely available at edgar.biocomp.unibo.it.

\section{Construction and content}

Data sources of associations between genes and diseases In order to collect a comprehensive resource of associations among genes and diseases we integrated data from OMIM (May 2016 release) [2], ClinVar (May 2016 release) [3] and Humsavar (June 2016 release) [4]. The primary accessions for genes are HGNC codes [20], while
OMIM identifiers are adopted to identify phenotypes. 2839 OMIM phenotype codes corresponding to identical or similar diseases, characterized by genetic heterogeneity, have been clustered into 357 phenotypic series, as defined by OMIM. Synonymic or alternative gene names were reduced to the HGNC gene primary codes, as reported in HGNC (June 2016 release).

On the overall, 5337, 4358 and 3365 gene-disease associations were collected from OMIM, ClinVar and Humsavar, respectively, by retaining only associations with unambiguous identification codes for both genes and diseases. After removing redundancy, the final dataset contains 5729 gene-disease associations, involving 3658 genes associated with 2672 diseases. These 2672 disease IDs correspond to 2315 OMIM IDs for phenotypes and 357 phenotypic series, or to 5154 when the 357 phenotypic series are brought back in 2839 OMIM IDs for phenotypes.

\section{Gene annotation}

All genes have been associated with the corresponding Ensembl codes (June 2016 version) [15] with BioMart [21]. Cytogenetic locations on the GrCh38 version of the human genome were therefrom derived. Out of 3658, 30 genes encode for microRNAs and tRNAs. For the 3628 protein coding genes, links to the SwissProt and PDB databases were also retrieved: all genes are linked to at least one SwissProt entry (for a total of 3718 entries) and 1682 genes are linked to at least one PDB entry (for a total of 14,578 PDB entries).

Functional annotation based on Gene Ontology (GO) terms was retrieved from GOOSE, the Online SQL Environment for $\mathrm{GO}$ terms implemented in the AmiGO2 portal [22]. All three GO sub-ontologies (Molecular Function: MF; Biological Process: BP; Cellular Component: CC) were considered. Given a GO term, the ancestor terms in the directed acyclic graph of $\mathrm{GO}$ (version 2.4) were retrieved by considering the relations "is a subtype of" and "part of". The information content (IC) was computed for each GO term, adopting standard methods [23], with the following equation:

$$
I C=-\log _{2}\left(\frac{N_{G O}}{N_{\text {root }}}\right)
$$

where $N_{G O}$ is the number of human genes endowed with the particular GO term and $N_{\text {root }}$ is the number of human genes annotated with all the terms of the considered subontology, as derived from GOOSE [22]. IC lower limit is zero; high IC values indicate that a small number of genes is annotated with a particular GO term in the human genome and therefore the annotation is highly informative. 
Associations with KEGG (version 77.0) [24] and REACTOME (version 53) [25] pathways were extracted from SwissProt.

Relationships among genes involved in the same disease eDGAR integrates several information in order to annotate the possible relationships among protein coding genes related to the same polygenic or heterogeneous disease. The following features are considered:

- Protein-protein interactions, as derived from the multimeric structures deposited at the PDB (February 2016 release) [16], from STRING (version 10.0) [26] and from the experimental data available in BIOGRID (version 3.4) [27]. From the human STRING network, we retained only high confidence links (score $\geq 0.7$ ) with annotated "action". Physical and genetic interactions of BIOGRID are reported separately. For all the considered human interactomes, eDGAR reports both direct and indirect interactions involving one intermediate gene. In addition, we supplemented data on interactions with selected annotations from manually curated features from SwissProt, including links to the PDB and the literature.

- Interactions in stable and functional complexes reported in the following resources: CORUM, listing 2837 mammalian complexes involving 3198 protein chains (16\% of the human protein-coding genes) [28], the soluble complex census, listing 622 complexes involving 3006 protein chains [29]. This last resource is referred in the following as CENSUS.

- Functional GO terms and KEGG/REACTOME pathways shared by at least two genes.

- Functional GO terms and KEGG/REACTOME pathways retrieved with NET-GE $[18,19]$, a network based tool that performs the statistically-validated enrichment analysis of sets of human genes by exploiting the human STRING interactome; a significance of $5 \%$ was considered when retrieving statistically enriched terms on the basis of the Bonferronicorrected $p$-values computed with NET-GE;

- Regulatory interactions derived from TRRUST [30], a curated database of interactions among 748 human transcription factors (TF) and 1975 non-TF targets. Given a set of genes associated with the same disease, eDGAR reports the presence of TF/target pairs and of groups of genes co-regulated by the same TF (belonging or not to the set);

- Co-localization in neighboring loci on the same chromosome: we highlighted genes located in the same cytogenetic band or in the tandem repeat regions listed in the DGD database [31]. DGD collects 945 groups consisting of 3543 genes in humans, likely deriving from duplications of ancestor genes.

\section{Database structure and visualization}

The database is implemented with PostgreSQL [32], an open source relational database system. Data stored in the database are retrieved using custom Python programs, while the output of the analysis is visualized in HTML pages using modern technologies like JavaScript. In particular, networks are encoded in JSON format and visualized using the JavaScript library D3.js [33]. We adopted a well known plug-in for jQuery called DataTables [34] for table visualizations, allowing the user to sort tables by columns and text-search inside each table.

\section{Results and discussion}

Statistics of the database content

The present release of eDGAR collects 5729 associations between 2672 diseases and 3658 different genes. Figure 1a plots the distribution of the number of genes associated with the same disease, which ranges from one (in 2051 monogenic diseases) to 69 (in the case of the "Retinitis pigmentosa" phenotypic series, OMIM: PS268000). The 621 diseases associated with multiple genes comprise both heterogeneous and polygenic diseases. On the overall, they account for 3678 associations with 2600 genes, 2576 of which code for proteins.

The database also shows a high level of pleiotropy (association of a single gene to several diseases) as shown in Fig. 1b. The most pleiotropic gene is FGFR3 that codes for the fibroblast growth factor receptor 3 and is associated with 16 different diseases.

\section{Statistics of gene annotation}

Table 1 lists major annotations of the 3658 genes related to diseases. All but 30 genes are coding for proteins reported in SwissProt; for $46.4 \%$ of them, structural information is available in PDB. Membrane proteins, transcription factors and enzymes account for 52\%, $7 \%$ and $31 \%$, respectively. Almost all the protein-coding genes are functionally annotated: the fraction of genes endowed with GO terms ranges from $94.2 \%$ to $98.6 \%$, depending on the sub-ontology (Molecular Function (MF), Biological Process (BP) and Cellular Component (CC)). A smaller percentage of genes are associated with KEGG and REACTOME pathways ( $56.7 \%$ and $62.8 \%$, respectively).

When considering human interactomes, $91.3 \%$ and 9.7\% of the genes are present in BIOGRID with physical and genetic interactions, respectively; for $82.5 \%$ of the genes, STRING reports high confidence interactions (score $\geq 0.7$ ). Some $20 \%$ of the genes encode for protein chains involved in functional complexes, as described in the CORUM and CENSUS collections. TRRUST lists some 1036 genes as part of the human regulatory 

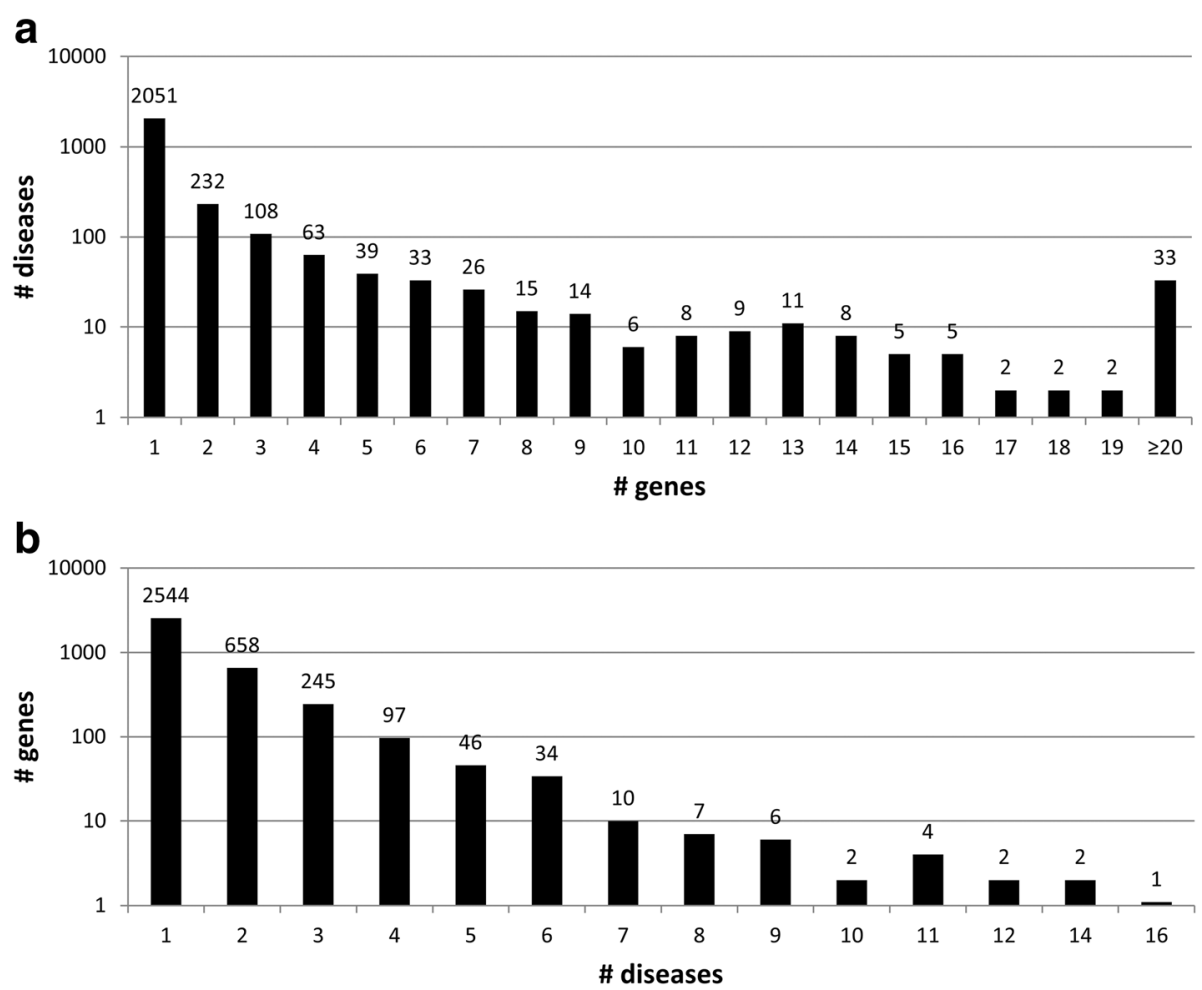

Fig. 1 Distribution of gene-disease associations. The Y-axis scale is logarithmic. a Number (\#) of genes associated with diseases. 2672 diseases are distributed with respect to the number of associated genes. 2051 diseases are monogenic; 621 diseases are associated with multiple genes (from 2 to 69 ). b Number (\#) of diseases associated to genes. 3658 genes are distributed with respect to the number of associated diseases. 2544 genes are associated with a single disease; 1114 genes are associated with multiple diseases (from 2 to 16)

network, of which 253 code for TFs and 783 are non-TF targets.

The level of annotation of the 2576 protein coding genes involved in heterogeneous or polygenic diseases is similar to that of all the genes collected in eDGAR.

Relations among genes associated with the same disease eDGAR lists the relations among different genes associated with the same multigenic disease (statistics is in Table 2). $21.9 \%$ of diseases involve at least one pair of genes located in the same cytogenetic band and in $8.2 \%$ of the cases, genes are tandem repeats originated by duplications. These genes are likely to undergo the same regulation mechanisms and to be coexpressed [33].

Many diseases involve at least one pair of genes directly linked in interactomes: $40.3 \%$ and $46.9 \%$, considering BIOGRID or STRING networks, respectively. The rates increase to $66.1 \%$ and $65.4 \%$ when considering also indirect interactions involving one intermediate gene not associated with the disease. $6.3 \%$ of diseases involve pairs of genes in a Transcription Factor (TF)/target relationship and $44 \%$ involve genes co-regulated by the same TF (considering also TFs not directly associated with the disease). The large majority of diseases (from $94.4 \%$ to $97.3 \%$, depending on the sub-ontology) is associated with at least one pair of genes sharing GO terms. More than $90 \%$ of all the possible pairs of genes involved in the same disease have common BP and CC terms; the percentage is somehow smaller (76\%) for MF sub-ontology. The total number of GO annotations shared by pairs of genes for BP, MF and CC is 72,787 (unique terms: 4582), 13,113 (unique terms: 915) and 16,298 (unique terms: 656), respectively. Overall, these data confirm the notion that genes associated with the same disease share some level of functional similarity, a view previously suggested for a small number of multigenic diseases [14]. However, being GO terms organized in a directed acyclic graph for each root, the information conveyed by the shared annotations can be very different, going from very general to very specific terms. The information content (IC, see Eq. 1) is routinely associated with GO terms in order to evaluate their specificity with respect of the available annotation of all human genes. The IC values of our dataset range from 0 (corresponding to the root $\mathrm{GO}$ term) to 10 (corresponding to the most specific terms). The average IC values for MF, BP and $\mathrm{CC}$ shared terms are $5.8 \pm 1.7,5.9 \pm 1.7$, and $5.8 \pm 1.9$, respectively. For each disease, the specificity of the annotation is evaluated by extracting the best IC values among the GO terms shared by pairs of co-associated genes (Fig. 2a). 
Table 1 Gene annotation in eDGAR

\begin{tabular}{|c|c|c|c|c|}
\hline & \multicolumn{2}{|l|}{ All diseases } & \multicolumn{2}{|c|}{ Diseases associated with multiple genes } \\
\hline & $\#$ genes $^{a}$ & \# associated diseases ${ }^{\mathrm{b}}$ & $\#$ genes $^{a}$ & $\#$ associated diseases $^{\mathrm{b}}$ \\
\hline Total number & 3658 & 2672 & 2600 & 621 \\
\hline Protein coding genes & $3628(100 \%)$ & $2655(100 \%)$ & $2576(100 \%)$ & $619(100 \%)$ \\
\hline with PDB entry & $1682(46.4 \%)$ & $1625(61.2 \%)$ & $1176(45.7 \%)$ & $512(82.7 \%)$ \\
\hline Membrane proteins & $1891(52.1 \%)$ & $1644(61.9 \%)$ & $1364(53.0 \%)$ & $517(83.5 \%)$ \\
\hline Enzymes (with E.C number) & $1112(30.7 \%)$ & 1045 (39.4\%) & $688(26.7 \%)$ & $363(58.6 \%)$ \\
\hline Reported in TRRUST (as TF) & $253(7.0 \%)$ & $358(13.5 \%)$ & 179 (6.9\%) & $157(25.4 \%)$ \\
\hline Reported in TRRUST (as target) & $783(21.6 \%)$ & $969(36.5 \%)$ & $570(22.1 \%)$ & $405(65.4 \%)$ \\
\hline Annotated with GO MF & 3419 (94.2\%) & 2575 (97.0\%) & 2419 (93.9\%) & 617 (99.7\%) \\
\hline Annotated with GO BP & $3538(97.5 \%)$ & 2619 (98.6\%) & $2514(97.6 \%)$ & $618(99.8 \%)$ \\
\hline Annotated with GO CC & $3576(98.6 \%)$ & $2644(99.6 \%)$ & $2533(98.3 \%)$ & 618 (99.8\%) \\
\hline Associated with KEGG pathways & 2057 (56.7\%) & $1868(70.4 \%)$ & $1430(55.5 \%)$ & $549(88.7 \%)$ \\
\hline Associated with REACTOME & $2278(62.8 \%)$ & 2007 (75.6\%) & 1595 (61.9\%) & $563(91.0 \%)$ \\
\hline With physical BIOGRID interactions & 3307 (91.3\%) & $2502(94.2 \%)$ & $2346(91.2 \%)$ & 609 (98.4\%) \\
\hline With genetic BIOGRID interactions & $351(9.7 \%)$ & $472(17.8 \%)$ & $259(10.1 \%)$ & $247(39.9 \%)$ \\
\hline With STRING interactions & $2992(82.5 \%)$ & $2341(88.2 \%)$ & $2146(83.3 \%)$ & 609 (98.4\%) \\
\hline Part of CORUM complexes & 714 (19.7\%) & $706(26.6 \%)$ & $558(21.7 \%)$ & 340 (54.9\%) \\
\hline Part of CENSUS complexes & $696(19.2 \%)$ & $689(26.0 \%)$ & $501(19.4 \%)$ & $296(47.8 \%)$ \\
\hline In tandem repeats & $381(10.5 \%)$ & $448(16.9 \%)$ & $280(10.9 \%)$ & $234(37.8 \%)$ \\
\hline
\end{tabular}

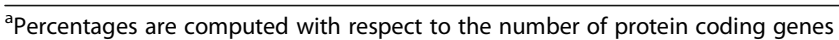

${ }^{b}$ Percentages are computed with respect to the number of diseases associated with protein coding genes

Table 2 Features shared by genes involved in the same heterogeneous or polygenic diseases

\begin{tabular}{|c|c|c|c|}
\hline & \# diseases & \# pairwise relations & \# protein coding genes \\
\hline Total number & 621 & 25,100 & 2576 \\
\hline \multicolumn{4}{|l|}{ With pairs of genes: } \\
\hline In same cytogenetic band & $136(21.9 \%)$ & $326(1.3 \%)$ & $335(13.0 \%)$ \\
\hline In tandem repeat & $51(8.2 \%)$ & $58(0.2 \%)$ & $92(3.6 \%)$ \\
\hline In TF/target pairs & $39(6.3 \%)$ & $81(0.3 \%)$ & $94(3.6 \%)$ \\
\hline Co-regulated by the same TF (not involved in the disease) & $273(44.0 \%)$ & $2308(9.2 \%)$ & $626(24.3 \%)$ \\
\hline Sharing MF GO & $586(94.4 \%)$ & $19,075(76.0 \%)$ & 2369 (92.0\%) \\
\hline Sharing BP GO & $597(96.1 \%)$ & $22,948(91.4 \%)$ & $2502(97.1 \%)$ \\
\hline Sharing CC GO & $604(97.3 \%)$ & $23,645(94.2 \%)$ & $2519(97.8 \%)$ \\
\hline Sharing KEGG pathway & $349(56.2 \%)$ & $3129(12.5 \%)$ & $1074(41.7 \%)$ \\
\hline Sharing REACTOME pathway & $474(76.3 \%)$ & $9806(39.1 \%)$ & $1554(60.3 \%)$ \\
\hline Interacting in PDB & $96(15.5 \%)$ & $207(0.8 \%)$ & $199(7.7 \%)$ \\
\hline In the same CORUM complex & $86(13.8 \%)$ & $469(1.9 \%)$ & $225(8.7 \%)$ \\
\hline In the same CENSUS complex & $45(7.2 \%)$ & $166(0.7 \%)$ & $119(4.6 \%)$ \\
\hline Directly linked in STRING & $291(46.9 \%)$ & $1535(6.1 \%)$ & $932(36.2 \%)$ \\
\hline Indirectly linked in STRING & $115(18.5 \%)$ & $4355(17.4 \%)$ & $1346(52.3 \%)$ \\
\hline Directly linked in BIOGRID (physical interaction) & $250(40.3 \%)$ & $944(3.8 \%)$ & $799(31.0 \%)$ \\
\hline Indirectly linked in BIOGRID (physical interaction) & $160(25.8 \%)$ & $5228(20.8 \%)$ & 1607 (62.4\%) \\
\hline Directly linked in BIOGRID (genetic interaction) & $9(1.4 \%)$ & $13(0.1 \%)$ & $19(0.7 \%)$ \\
\hline Indirectly linked in BIOGRID (genetic interaction) & $25(4.0 \%)$ & $45(0.2 \%)$ & $62(2.4 \%)$ \\
\hline
\end{tabular}



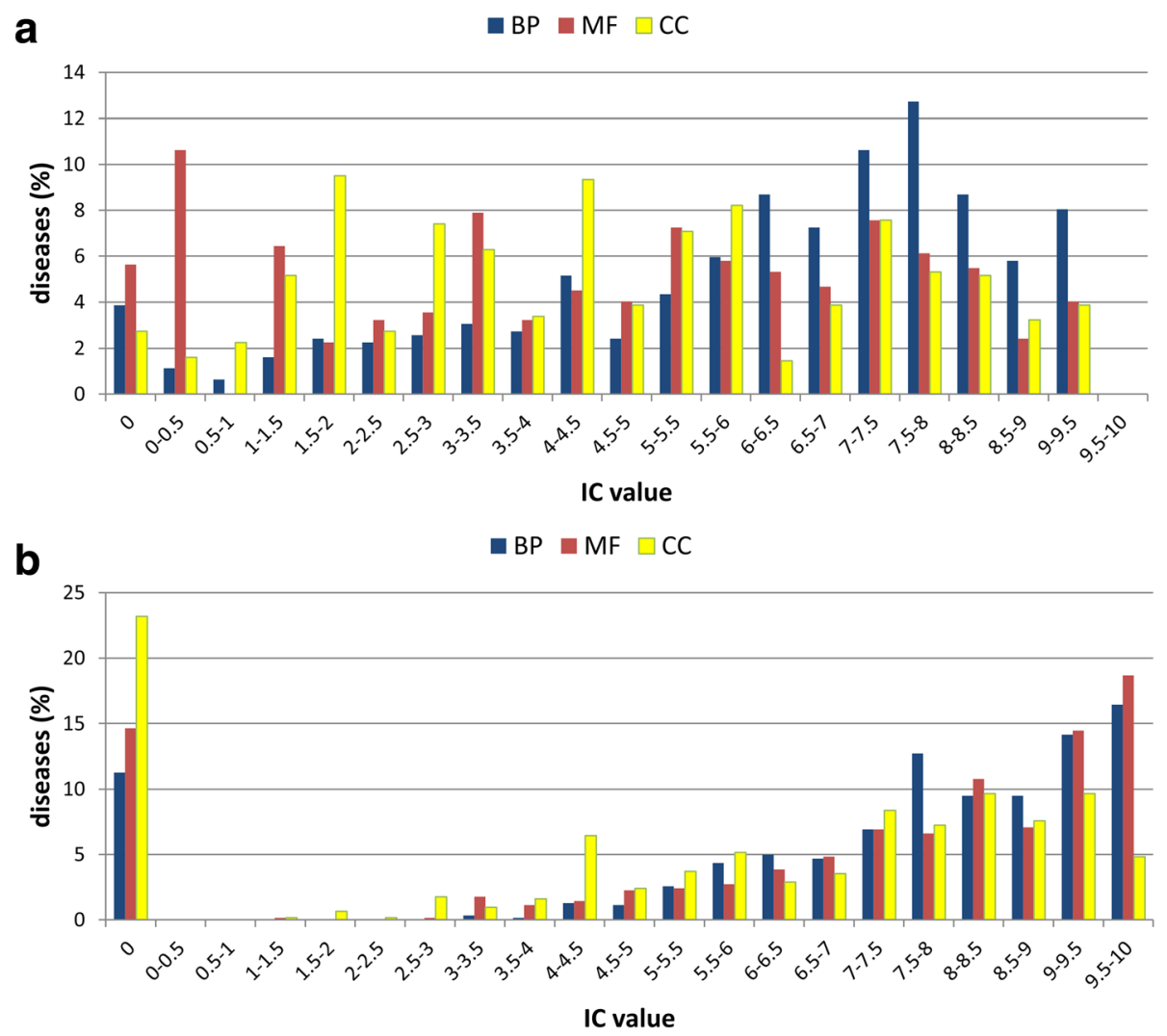

Fig. 2 Distribution of best IC values of GO terms for genes involved in multigenic diseases. a GO terms shared by genes; $\mathbf{b} \mathrm{GO}$ terms after enrichment with NET-GE. For each multigenic disease, IC values of gene-associated GO terms (of the three different roots) are evaluated (Eq. 1). In the figure, the highest IC for each disease is shown. The frequency is computed with respect to the total number of multigenic diseases (621). When $I C=0$, genes associated with multigenic disease do not share or enrich $G O$ terms (panel $\mathbf{a}$ and $\mathbf{b}$ respectively)

For all the sub-ontologies, the best IC values are very spread, and it is evident that on average the most specific terms (highest IC values) belong to the BP subontology: genes pairs sharing $\mathrm{BP}, \mathrm{MF}$ and $\mathrm{CC}$ terms with IC $\geq 5$ are present in $72 \%, 49 \%$ and $46 \%$ of the diseases, respectively (see Fig. 2a). When a different distribution based on a median is adopted, the pattern is very similar (Additional file 1: Fig. S1A). Genes involved in the same disease share also KEGG and REACTOME pathways (56.2\% and $76.3 \%$, respectively (Table 2 )).

\section{NET-GE enrichment}

In order to better highlight functions shared by groups of genes associated with the same disease, we adopt NET-GE $[18,19]$, our recently developed network based tool for functional enrichment. For each functional sets of GO terms and/or KEGG or REACTOME pathways, NET-GE builds a network containing all the human genes annotated with the terms (seeds) and including all the connecting genes (the reference human interactome is derived from STRING). Input genes are mapped into the pre-computed NET-GE networks and enrichment analysis is performed. Outputs are Bonferroni-corrected $p$-values, measuring the overrepresentation of each term in the input set. Due to its network-based nature, NETGE can enrich terms not present in the list of annotations of the input set. Table 3 lists the results of NETGE on the groups of genes associated with the same disease, considering a $5 \%$ significance. For the majority of diseases, NET-GE enriches GO terms of the three subontologies and pathways of KEGG and REACTOME. BP is the sub-ontology type most frequently enriched. The total number of GO annotations enriched for heterogeneous and polygenic diseases is 17,029, 4851 and 3910 (Table 3, rightmost column), with average IC values $6.1 \pm 1.8,7.1 \pm 2$, and $6.4 \pm 2$ for $\mathrm{BP}, \mathrm{MF}$ and $\mathrm{CC}$

Table 3 NET-GE functional enrichment of groups of genes involved in the same disease

\begin{tabular}{lll}
\hline & \# diseases & \# annotations \\
\hline KEGG pathways & $412(66.3 \%)$ & 2753 \\
REACTOME pathways & $488(78.6 \%)$ & 4130 \\
GO MF terms & $530(85.3 \%)$ & 4851 \\
GO BP terms & $551(88.7 \%)$ & 17,029 \\
GO CC terms & $477(76.8 \%)$ & 3910 \\
\hline
\end{tabular}


respectively (Fig. 2b, reporting the distribution of the best IC values among the terms enriched for each disease; for a different distribution based on IC median values, see Additional file 1: Figure S1B).

\section{The user interface}

eDGAR is publicly available as a web server at edgar.biocomp.unibo.it with browsing and search options. Browsing is performed with the "Main Table" page that contains all the collected associations between genes and diseases, along with the indication of source databases.

The Search engine allows to access the database with different identifiers: HGNC symbols and Ensembl identifiers for genes, UniProt accession for proteins, OMIM identifiers or disease names for phenotypes and phenotypic series. The user may also search with a set of genes and retrieve shared annotation features.

Two types of pages can be visualized: i) gene specific pages, reporting the associations to diseases and the available gene annotations; ii) disease specific pages, reporting the associations with genes and, in case of heterogeneous and polygenic diseases, the list of relationships linking the different genes, organized into different tables. Interactions from STRING, PDB, BIOGRID, CORUM, CENSUS can also be visualized by means of graphs, reporting direct and indirect interactions. The graphs show the gene associated with the disease as blue nodes and other genes in interactions as pale blue nodes; the direct interactions are visualized as green edges and the indirect interactions as thin black edges (see Fig. 3). Clicking on a node, the user is redirected to the correspondent gene page.

\section{A case study: Hypoparathyroidism}

Hypoparathyroidism (OMIM 146200) is an endocrine deficiency disease characterized by low serum calcium levels, elevated serum phosphorus levels and absent or low levels of parathyroid hormone (PTH) in blood [35]. The metabolism of the patient may be altered: the vitamin $\mathrm{D}$ supply is inadequate and the magnesium metabolism is irregular. In some clinical panel, hypocalcemia can lead to dramatic effects such as tetany, seizures, altered mental status, refractory congestive heart failure, or stridor.

In eDGAR the familial isolated hypoparathyroidism (OMIM 146200) is associated with three different genes: GCM2 and PTH (both reported in OMIM, ClinVar and Humsavar) and CASR (reported only in ClinVar). CASR is an extracellular calcium-sensing receptor whose activity is mediated by G-proteins, PTH is the parathyroid hormone, whose function is to increase calcium level both by promoting the solution of bone salts and by preventing their renal excretion, and GCM2 (Glial cell missing homolog 2) is a probable transcriptional regulator, considering the SwissProt annotation. The "Transcription Factor (TF) annotation from TRRUST" table in eDGAR reports that GMC2 is a TF that regulates the expression of both PTH and CASR. Moreover, when considering "Interactions from STRING" table, PTH and CASR are in direct interaction, labelled as "binding" and "expression". The shared BP GO terms with the highest IC values are "response to vitamin D" and "response to fibroblast growth factor", both involving CASR and PTH. The response to vitamin $\mathrm{D}$, whose metabolism is often altered in hypoparathyroidism, and a strict interplay between fibroblast growth factors and parathyroid hormone have been previously reported [36-38]. PTH and CASR are also involved in the same REACTOME pathways related to GPCR ligand binding and signaling. No shared KEGG term is found.

NET-GE enrichment for BP for the three genes include new terms endowed with high IC values, like "regulation of amino acid transport", "negative regulation of muscle contraction". Some of these new annotations are related to the severe symptoms of hypothyroidisms, namely tetany and seizure. NET-GE allows retrieving enriched KEGG pathways, such as "Circadian entrainment (hsa04713)", "Inflammatory mediator regulation of TRP channels (hsa04750)", "Gap junction (hsa04540)" and "Insulin secretion (hsa04911)". None of the three genes is directly involved in the four pathways; PTH and CASR are part of the networks defined by NETGE exploiting the STRING network. Interestingly, these new annotations highlight previously reported impairments of both circadian rhythms impairment and insulin secretion associated with hypoparathyroidism $[39,40]$.

Figure 3 reports a summary of the information provided by eDGAR for hypothyroidism (OMIM 146200), showing how it allows to collect the different types of relations among the involved genes in a unique page integrating data from many resources.

\section{Conclusions}

eDGAR is a resource for the study of the associations between genes and diseases. It collects 2672 diseases, associated with 3658 different genes, for a total number of 5729 gene-disease associations. The novelty of eDGAR is the integration of different sources of gene annotation and in particular, for the 621 heterogeneous/polygenic diseases, eDGAR offers the possibility of analyzing functional and structural relations among co-involved genes. We provide direct interactions between pairs of genes (reported in STRING or BIOGRID) for 291 diseases and indirect interactions for some other 250 diseases. For 273 diseases, at least 


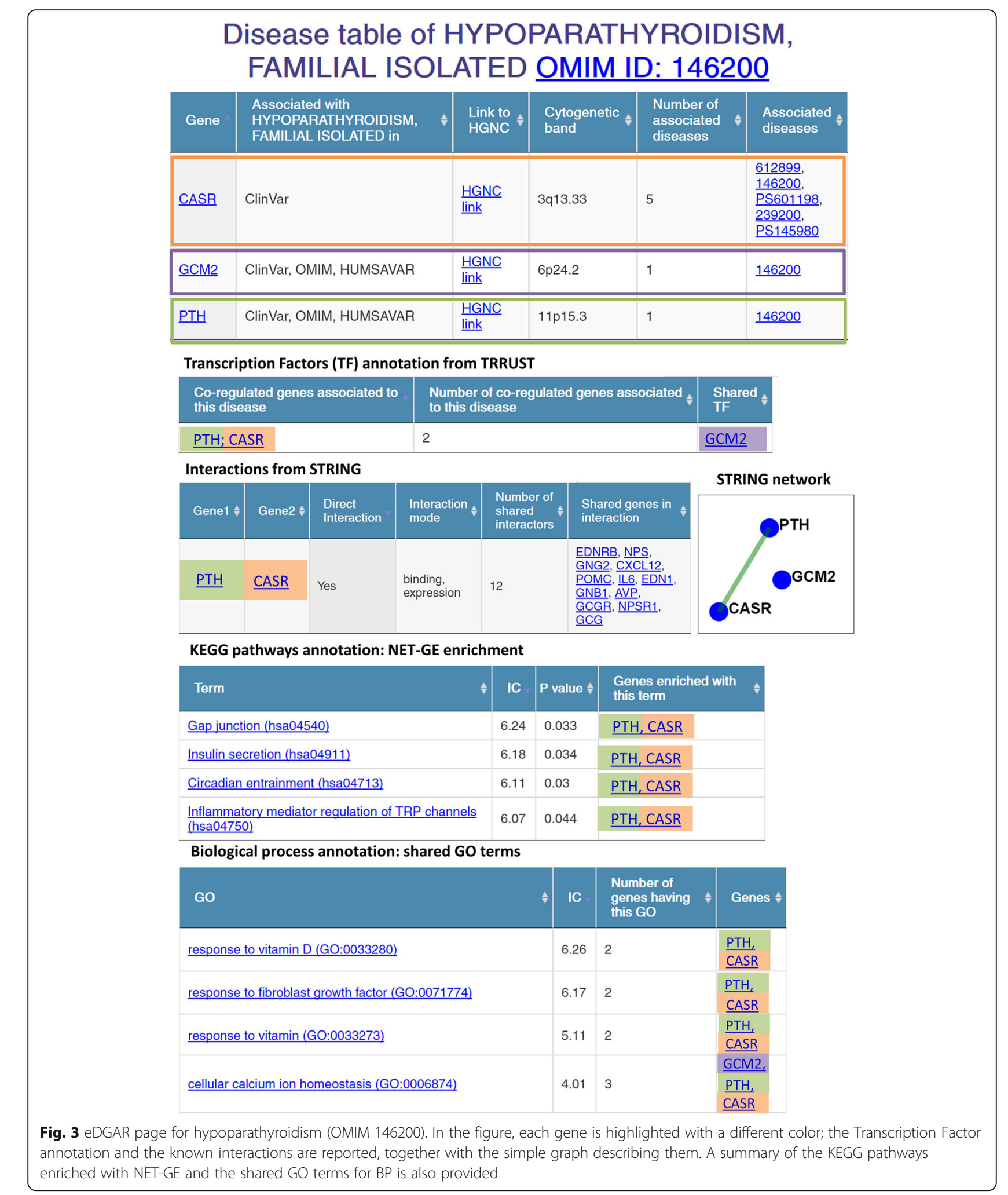

one pair of genes is under regulatory interaction of the same TF, while 39 disease are associated with genes being a TF/target couple. For 612 diseases, at least one pair of genes share GO terms and/or
KEGG/REACTOME pathways. In particular, genes involved in the same disease most frequently share terms of the BP sub-ontology. This is confirmed also when analyzing the statistically significant functional 
terms enriched with NET-GE for 606 diseases. The relations among genes involved in the same disease are often complex and different pairs of genes are linked in different ways. eDGAR is a resource for better tackling the complexity of gene interactions at the basis of multigenic diseases. The database will be updated following the major releases of the different underlying data resources at least once a year.

\section{Additional file}

Additional file 1: Figure S1. Distribution of median IC values of $G O$ terms for genes involved in multigenic diseases. A: GO terms shared by genes; B: GO terms enriched with NET-GE. For each multigenic disease, IC value of gene-associated $\mathrm{GO}$ terms (of the three different roots) are evaluated (Eq. 1). In the figure the median IC for each disease is shown. The frequency is computed with respect to the total number of multigenic diseases (621). When $I C=0$, genes associated with multigenic disease do not share or enrich $\mathrm{GO}$ terms (panel A and B respectively). (PNG $393 \mathrm{~kb}$ )

\section{Acknowledgements}

Not applicable.

\section{Funding}

Publication costs for this article were provided by PRIN 2010-2011 project 20108XYHJS (to P.L.M.) (Italian MIUR); COST BMBS Action TD1101 and Action BM1405 (European Union RTD Framework Program, to R.C); PON projects PON01_02249 and PAN Lab PONa3_00166 (Italian Miur to R.C. and P.L.M.); FARB UNIBO 2012 (to R.C.)

\section{Availability of data and materials}

The dataset generated during the current study is available and downloadable at edgar.biocomp.unibo.it.

\section{Authors' contributions}

$\mathrm{RC}, \mathrm{PLM}$, and GB conceived and designed the work and wrote the paper. GB collected and curated data. SB ran the NET-GE predictions. GB, GP, and CS implemented the web server. PLM, GB and RC analysed and interpreted data on disease related variations. All authors critically revised and approved the manuscript.

\section{Ethics approval and consent to participate}

The authors declare that they used only public data.

\section{Consent for publication}

Not applicable.

\section{Competing interests}

The authors declare that they have no competing interests.

\section{Author details}

'Biocomputing Group, BiGeA, University of Bologna, Bologna, Italy. ${ }^{2}$ Interdepartmental Center «Giorgio Prodi» for Cancer Research, University of Bologna, Bologna, Italy.

\section{Published: 10 August 2017}

\section{References}

1. Kann MG. Advances in translational bioinformatics: computational approaches for the hunting of disease genes. Brief Bioinform. 2010;11(1):96-110

2. Amberger JS, Bocchini CA, Schiettecatte F, Scott AF, Hamosh A. OMIM.Org: online Mendelian inheritance in man (OMIM)), an online catalog of human genes and genetic disorders. Nucleic Acids Res. 2015;43(Database issue): D789-98.
3. Landrum MJ, Lee JM, Benson M, Brown G, Chao C, Chitipiralla S, Gu B, Hart J, Hoffman D, Hoover J, Jang W, Katz K, Ovetsky M, Riley G, Sethi A, Tully R, Villamarin-Salomon R, Rubinstein W, Maglott DR. ClinVar: public archive of interpretations of clinically relevant variants. Nucleic Acids Res. 2016;44(D1):D862-8.

4. UniProt Consortium. UniProt: a hub for protein information. Nucleic Acids Res. 2015;43(Database issue):D204-12.

5. Stenson PD, Ball EV, Mort M, Phillips AD, Shaw K, Cooper DN. The Human Gene Mutation Database (HGMD) and its exploitation in the fields of personalized genomics and molecular evolution. Curr Protoc Bioinformatics. 2012;39:1.13:1.13.1-1.13.20.

6. Piñero J, Bravo À, Queralt-Rosinach N, Gutiérrez-Sacristán A, Deu-Pons J, Centeno E, García-García J, Sanz F, Furlong LI. DisGeNET: a comprehensive platform integrating information on human disease-associated genes and variants. Nucl Acids Res. 2016:45(D1):D833-9.

7. Rappaport N, Twik M, Plaschkes I, Nudel R, Stein TI, Levitt J, Gershoni M, Morrey CP, Safran M. Lancet D; MalaCards: an amalgamated human disease compendium with diverse clinical and genetic annotation and structured search. Nucl Acids Res. 2016:45(D1):D877-87.

8. Gazzo AM, Daneels D, Cilia E, Bonduelle M, Abramowicz M, Van Dooren S, Smits G, Lenaerts T. DIDA: a curated and annotated digenic diseases database. Nucleic Acids Res. 2016;44(D1):D900-7.

9. McClellan J, King MC. Genetic heterogeneity in human disease. Cell. 2010;141(2):210-7.

10. Weeks DE, Lathrop GM. Polygenic disease: methods for mapping complex disease traits. Trends Genet. 1995;11(12):513-9.

11. Fu W, O'Connor TD, Akey JM. Genetic architecture of quantitative traits and complex diseases. Curr Opin Genet Dev. 2013;23(6):678-83.

12. Cardon $L R$, Harris T. Precision medicine, genomics and drug discovery. Hum Mol Genet. 2016;25(R2):R166-72.

13. Goh Kl, Cusick ME, Valle D, Childs B, Vidal M, Barabási AL. The human disease network. Proc Natl Acad Sci U S A. 2007:104(21):8685-90.

14. Oti M, Brunner H. The modular nature of genetic diseases. Clin Genet. 2007;71:1-11.

15. Aken BL, Ayling S, Barrell D, Clarke L, Curwen V, Fairley S, Fernandez Banet J, Billis K, García Girón C, Hourlier T, Howe K, Kähäri A, Kokocinski F, Martin FJ, Murphy DN, Nag R, Ruffier M, Schuster M, Tang YA, Vogel JH, White S, Zadissa A, Flicek P, Searle SM. The Ensembl gene annotation system. Database (Oxford). 2016; pii: baw093.

16. Berman HM, Westbrook J, Feng Z, Gilliland G, Bhat TN, Weissig H, Shindyalov IN. Bourne PE the protein data bank. Nucleic Acids Res. 2000;28:235-42.

17. The Gene Ontology Consortium.. Expansion of the Gene Ontology knowledgebase and resources. Nucleic Acids Res. 2016. pii: gkw1108.

18. Di Lena P, Martelli PL, Fariselli P. Casadio R. NET-GE: a novel NETwork-based Gene Enrichment for detecting biological processes associated to Mendelian diseases. BMC Genomics. 2015;16(Suppl 8):S6.

19. Bovo S, Di Lena P, Martelli PL, Fariselli P, Casadio R. NET-GE: a webserver for NETwork-based human gene enrichment. Bioinformatics. 2016;32(22):3489-91.

20. Yates B, Braschi B, Gray KA, Seal RL, Tweedie S, Bruford EA. Genenames.org: the HGNC and VGNC resources in 2017. Nucleic Acids Res. 2016. pii: gkw1033.

21. Kasprzyk A. BioMart: driving a paradigm change in biological data management. Database (Oxford). 2011:bar049.

22. Munoz-Torres M, Carbon S. Get GO! Retrieving GO data using AmiGO, QuickGO, API, files, and tools. Methods Mol Biol. 2017:1446:149-60.

23. Shannon CE. A mathematical theory of communication. Bell Syst Techn J. 1948:27:379-423.

24. Kanehisa M, Sato Y, Kawashima M, Furumichi M, Tanabe M. KEGG as a reference resource for gene and protein annotation. Nucleic Acids Res. 2016:44(D1):D457-62.

25. Fabregat A, Sidiropoulos K, Garapati P, Gillespie M, Hausmann K, Haw R, Jassal B, Jupe S, Korninger F, McKay S, Matthews L, May B, Milacic M, Rothfels K, Shamovsky V, Webber M, Weiser J, Williams M, Wu G, Stein L, Hermjakob H, D'Eustachio P. The Reactome pathway knowledgebase. Nucleic Acids Res. 2016;44(D1):D481-7.

26. Szklarczyk D, Franceschini A, Wyder S, Forslund K, Heller D, Huerta-Cepas J, Simonovic M, Roth A, Santos A, Tsafou KP, Kuhn M, Bork P, Jensen L, von Mering C. STRING v10: protein-protein interaction networks, integrated over the tree of life. Nucleic Acids Res. 2015;43(Database issue):D447-52. 
27. Chatr-Aryamontri A, Breitkreutz BJ, Oughtred R, Boucher L, Heinicke S, Chen D, Stark C, Breitkreutz A, Kolas N, O'Donnell L, Reguly T, Nixon J, Ramage L, Winter A, Sellam A, Chang C, Hirschman J, Theesfeld C, Rust J, Livstone MS, Dolinski K, Tyers M. The BioGRID interaction database: 2015 update. Nucleic Acids Res. 2015;43(Database issue):D470-8.

28. Ruepp A, Waegele B, Lechner M, Brauner B, Dunger-Kaltenbach I, Fobo G, Frishman G, Montrone C, Mewes HW. CORUM: the comprehensive resource of mammalian protein complexes_-2009. Nucleic Acids Res. 2010;38(Database issue):D497-501.

29. Havugimana PC, Hart GT, Nepusz T, Yang H, Turinsky AL, Li Z, Wang PI, Boutz DR, Fong V, Phanse S, Babu M, Craig SA, Hu P, Wan C, Vlasblom J, Dar VU, Bezginov A, Clark GW, Wu GC, Wodak SJ, Tillier ER, Paccanaro A, Marcotte EM, Emili A. A census of human soluble protein complexes. Cell. 2012;150(5):1068-81.

30. Han H, Shim H, Shin D, Shim JE, Ko Y, Shin J, Kim H, Cho A, Kim E, Lee T, Kim H, Kim K, Yang S, Bae D, Yun A, Kim S, Kim CY, Cho HJ, Kang B, Shin S, Lee I. TRRUST: a reference database of human transcriptional regulatory interactions. Sci Rep. 2015;5:11432.

31. Ouedraogo M, Bettembourg C, Bretaudeau A, Sallou O, Diot C, Demeure O, Lecerf $F$. The duplicated genes database: identification and functional annotation of co-localised duplicated genes across genomes. PLoS One. 2012:7(11):e50653.

32. PostgreSQL. https://www.postgresql.org/. Accessed 1 December 2016.

33. Data-Driven. Documents. https://d3js.org/. Accessed 1 December 2016.

34. DataTables. https://datatables.net/. Accessed 1 December 2016.

35. Bilezikian J, Khan A, Potts J, et al. Hypoparathyroidism in the adult: epidemiology, diagnosis, pathophysiology, target organ involvement, treatment, and challenges for future research. J Bone Miner Res. 2011;26(10):2317-37.

36. Lai $Y$, Wang H, Xia $X$, Wang Z, Fan C, Wang $H$, Zhang H, Ding S, Teng W Shan Z. Serum fibroblast growth factor 19 is decreased in patients with overt hypothyroidism and subclinical hypothyroidism. Medicine (Baltimore). 2016;95(39)::5001.

37. Domouzoglou EM, Fisher FM, Astapova I, Fox EC, Kharitonenkov A, Flier JS, Hollenberg AN, Maratos-Flier E. Fibroblast growth factor 21 and thyroid hormone show mutual regulatory dependency but have independent actions in vivo. Endocrinology. 2014;155(5):2031-40.

38. Lee Y, Park YJ, Ahn HY, Lim JA, Park KU, Choi SH, Park DJ, Oh BC, Jang HC, Yi KH. Plasma FGF21 levels are increased in patients with hypothyroidism independently of lipid profile. Endocr J. 2013;60(8):977-83.

39. Bauer MS, Soloway A, Dratman MB, Kreider M. Effects of hypothyroidism on rat circadian activity and temperature rhythms and their response to light. Biol Psychiatry. 1992;32(5):411-25.

40. Yang N, Yao Z, Miao L, Liu J, Gao X, Fan H, Hu Y, Zhang H, Xu Y, Qu A, Wang G. Novel clinical evidence of an association between Homocysteine and insulin resistance in patients with hypothyroidism or subclinical hypothyroidism. PLoS One. 2015;10(5):e0125922.

\section{Submit your next manuscript to BioMed Central and we will help you at every step:}

- We accept pre-submission inquiries

- Our selector tool helps you to find the most relevant journal

- We provide round the clock customer support

- Convenient online submission

- Thorough peer review

- Inclusion in PubMed and all major indexing services

- Maximum visibility for your research

Submit your manuscript at www.biomedcentral.com/submit

) Biomed Central 